\title{
Leberschäden durch pflanzliche Medikamente und Nahrungsergänzungsmittel
}

Felix Stickel

\section{Was ist neu?}

- Absatz und Regulierung: Pflanzliche Medikamente und Nahrungsergänzungsmittel werden zunehmend häufig und weitgehend unkontrolliert konsumiert. Derzeit wird eine europaweite Regelung zur Registrierung pflanzlicher Medikamente und Nahrungsergänzungsmittel erarbeitet.

- Steigende Inzidenz: Leberschäden durch pflanzliche Medikamente und Nahrungsergänzungsmittel werden zunehmend häufiger beobachtet.

- Diagnosestellung: Die Diagnosestellung beruht in hohem Maße auf klinischem Verdacht und dem Ausschluss anderer Ursachen. Dabei können Kausalitäts-Scores hilfreich sein.

- Wissenschaftlich dokumentiertes Potenzial für Leberschäden: Prominente Beispiele für Leberschäden durch pflanzliche Medikamente und Nahrungsergänzungsmittel sind manche chinesische Kräutergemische, Produkte aus Kava, Schöllkraut und Grüntee.

- Nahrungsergänzungspräparate zur Gewichtsreduktion: In letzter Zeit werden besonders häufig Leberschäden bei Präparaten zur Gewichtsreduktion und zur Steigerung der sportlichen Fitness beobachtet.
Regelung zur Registrierung pflanzlicher Medikamente und Nahrungsergänzungsmittel gearbeitet, bei der die Europäische Arzneimittel-Agentur EMA Richtlinien der Good Agricultural Practice (GAP) und Good Manufacturing Practice (GMP) überwachen und Verstöße dagegen sanktionieren soll [3].

\section{Klinische Relevanz \\ Die Wahrscheinlichkeit, dass Patienten pflanzliche Medikamente bzw. Nahrungs- ergänzungsmittel einnehmen ist relativ hoch, sodass gezielt und wiederholt nachgefragt werden sollte, wenn es um die Ursachensuche bei unerklärten Leberschäden geht. Die Tatsache, dass pflanzliche Medikamente und Nahrungsergänzungsmittel verkäuflich sind, belegt nicht ihre Unbedenklichkeit.}

\section{Steigende Inzidenz}

Während die tatsächliche Inzidenz von Leberschäden durch pflanzliche Medikamente und Nahrungsergänzungsmittel nicht sicher zu erfassen ist, deuten mehrere Studien auf eine zunehmende Häufigkeit derartiger „medikamentös-bedingte Leberschäden (drug-induced liver injury - DILI)“ hin. Dabei sei betont, dass in einem erheblichen Prozentsatz auch schwerwiegende Zwischenfälle mit Todesfolge und der Notwendigkeit einer Lebertransplantation registriert wurden [4].

\section{Klinische Relevanz}

Leberschäden durch pflanzliche Medikamente und Nahrungsergänzungsmittel können erheblich sein und neben maximalen Therapiemaßnahmen wie Lebertransplantation auch tödliche Verläufe zur Folge haben.

\section{Diagnosestellung}

Die Zuordnung eines festgestellten Leberschadens zur Einnahme von pflanzlichen Medikamenten bzw. Nahrungsergänzungsmitteln ist nicht immer einfach. Sie erfordert zuerst einmal den klinischen Verdacht, dass dies die Ursache sein könnte. Häufig berichten Patienten nicht spontan über die Einnahme von pflanzlichen Medikamenten bzw. Nahrungsergänzungsmitteln, da sie diese nicht für wichtig halten, oder die Kritik des Arztes fürchten. 
Daher muss gezielt und manchmal wiederholt nachgefragt werden. Affirmative diagnostische Tests existieren nicht, wie bei den meisten synthetischen Medikamenten auch, sodass der Ausschluss anderer, durch Tests erfassbarer Ursachen ein zentraler Baustein der Diagnostik ist. Dabei können sogenannte Kausalitäts-Scores hilfreich sein, die jedoch nicht für DILI durch pflanzliche Medikamente und Nahrungsergänzungsmittel validiert sind [5, 6]. Unumgänglich aber ist eine sorgfältige Literaturrecherche nach entsprechenden Beschreibungen ähnlicher Fälle in entsprechenden Datenbanken.

\section{Klinische Relevanz}

Schlüssel zur Diagnosestellung ist der klinische Verdacht, eine sorgfältige Ausschlussdiagnose und Literaturrecherche sowie die Anwendung von Kausalitäts-Scores.

\section{Wissenschaftlich dokumentiertes Potenzial für Leberschäden}

Vielfach beschrieben sind Leberschäden im Zusammenhang mit dem Konsum von Pflanzen und deren Extrakten, die Pyrrolizidinalkaloide (PA) enthalten. Diese sind streng reguliert und dürfen in Deutschland z.B. nur noch zur äußerlichen Anwendung eingesetzt werden $(\checkmark$ Tab. 1$)$. Ein bekannter Vertreter ist Beinwell (Symphytum officinale), das nur noch als Salbe oder Creme gegen Muskel- und Gelenkschmerzen verwendet werden darf, da es bei oraler Einnahme ein sinusoidales Obstruktionssyndrom (früher: venookklusive Erkrankung) auslösen kann [7]. Zahlreiche andere Pflanzen in Europa enthalten ebenfalls PA, sodass die Möglichkeit einer Intoxikation damit nicht ausgeschlossen werden kann.

\section{Produkt/Wirkstoff \\ Pyrrolizidinalkaloide-haltige \\ Präparate (Symphytum, Heliotropium, Crotalaria, Senecio) \\ Jin Bu Huan \\ (Lypocodium serratum)}

TCM-Kombi mit Pfingstrose

(Paeonia officinalis) und weißem Diptam (Dictamnus dasycarpus)

„Shou-Wu Pian“ (Polygonum multiflorum)

Breynia officinalis

Chaparral

(Larrea tridentata)

Actractylis gummifera

Impila

(Callilepsis laureola)

Pennyroyal (Mentha pulegium)

Schöllkraut

(Chelidonium majus)

Kava (Piper methysticum)

Traubensilberkerze

(Actaea/Cimifuga racemosa)

Senna (Cassia angustifolia, Cascara sagrada)

Noni juice

(Morinda citrifolia)

Glucosamin/Chondroitinsulfat

Gotu Kola (Centella asiatica)

\section{Anwendungsbereich}

Keine klare Indikation. Kontamination von Getreide, Salat oder Kräutermischungen; Wundheilung (Beinwell)

Stimmungsaufhellung, Sedation

Atopische Dermatitis

u.a. Lebertonikum

Zahlreiche Indikationen

Arthralgien, Gewichtsreduktion, Antioxidans

Zahlreiche Anwendungen (Diuretikum, Kaugummi etc.)

Zahlreiche Indikationen

Abtreibungsmittel

Dyspepsie

Angststörungen, innere Unruhe

Postmenopausale Beschwerden

Laxativum

„Immunostimulation”, Gesundheitstonikum

Gelenkschmerzen

Gesundheitstonikum

\section{Leberschaden}

Sinusoidales Obstruktions-

syndrom (venookklusive

Erkankung)

Akute und chronische Hepatitis

Akute und fulminante Hepatitis

Akute und fulminante Hepatitis

Akute Hepatitis

Cholestatische Hepatitis, akutes Leberversagen

Akute Hepatitis

Akute Leberzellnekrose mit mikrovesikulärer Verfettung (ähnlich dem Reye-Syndrom)

Akute Leberzellnekrose

Cholestatische hepatitis, z. T. mit Autoantikörpern

Akute und fulminante Hepatitis

Akute und fulminante Hepatitis, autoimmune Phänomene

Cholestatische Hepatitis

Akute und fulminante Hepatitis

Akute autoimmune Hepatitis

Akute und chronische Hepatitis
Nicht bekannt

Tab. 1 Übersicht von pflanzlichen Medikamenten und Nahrungsergänzungsmitteln, die mit Leberschäden in Verbindung gebracht werden.

\section{Schädigungsmechanismus}

Dosis-bedingte Schädigung von Gefäßendothelien mit Okklusion der Sinusoide; Toxin: Pyrrolverbindungen

Nicht bekannt

Nicht bekannt

Nicht bekannt

Hemmung von Cyclooxygenase und Zytochrom P-450-Enzymen durch Nordihydroguajaretsäure

Nicht bekannt

Mitochondrienschaden durch Hemmung der oxidativen Phosphorylierung

Menthofurantoxizität

Nicht bekannt

Nicht bekannt; CYP 2D6 Polymorphismus?

Apoptose durch Mitochondrienschädigung

Autoimmunität durch Anthrachinone?

Nicht bekannt

Nicht bekannt

Nicht bekannt 
Tab. 2 Übersicht von pflanzlichen Präparaten zur Gewichtsreduktion, die mit Leberschäden assoziiert sind.
In der Traditionellen Chinesischen Medizin (TCM) werden über 13000 chinesische Kräuterkombinationen eingesetzt, viele davon seit mehreren Tausend Jahren. Dahre hält man ihren Einsatz für unbedenklich. Allerdings sind zahlreiche Kombinationen mit z.T. schweren Leberschäden in Verbindung gebracht worden. Eine systematische Ursachensuche gestaltet sich oft sehr schwierig, da die Präparate aus Gemischen bestehen, unterschiedliche Wirkstoffkonzentrationen enthalten und manchmal durch Toxine oder absichtlich zugemischte synthetische Arzneimittel kontaminiert sind (ausführlicher Überblick in [8]).

Gamander wurde in den frühen 90er-Jahren in Frankreich zur Unterstützung von Gewichtsreduktion zugelassen. In der Folge kam es zu einer großen Serie akuter, chronischer und auch fulminanter Leberschäden, die zum Zulassungsentzug führten. Ursächlich war die toxische Wirkung von darin enthaltenen Neo-Clerodan-Diterpenoiden, die durch eine Mitochondrienschädigung Leberzellapoptose auslösen [9].

Produkte mit Schöllkraut-Extrakt wurden zur Behandlung von dyspeptischen Beschwerden eingesetzt, ohne dass die Wirksamkeit durch klinische Evidenz belegt war. In den letzten Jahren sind über 100 Berichte von akuten Leberschäden an die Vigisearch Database der WHO in Uppsala gemeldet worden, sodass die WHO das Nutzen-RisikoVerhältnis als negativ beurteilt [10]. In Deutschland dürfen nach dieser Bewertung Präparate mit > 2,5 mg Chelidonin, einem Schöllkrautalkaloid, nicht mehr vertrieben werden. Der eigentliche Toxizitätsmechanismus ist jedoch nicht bekannt.
Zahlreiche Ereignisse mit z.T. schweren Leberschäden im Zusammenhang mit der Einnahme pflanzlicher Anxiolytika und Antidepressiva aus Extrakten des Rauschpfeffers (Kava; Piper methysticum rhizoma) führten 2003 zum Zulassungsentzug aller Kava-haltiger Präparate in Deutschland, der EU und Nordamerika. Meist kam es nach unterschiedlicher Einnahmedauer zu cholestatischen Hepatitiden, aber bei einigen Patienten auch zum fulminanten Leberversagen mit Lebertransplantation [11]. Auch bei Kava ist der Schädigungsmechanismus nicht abschließend geklärt.

Grüner Tee aus Camellia sinensis wird auf der ganzen Welt konsumiert und gilt als sehr bekömmlich. So überraschte es sehr, dass Präparate, die Grüntee-Extrakte enthielten, mit Leberschäden in Verbindung gebracht wurden. Eine systematische Analyse bewertete den Zusammenhang in den meisten Fällen als „wahrscheinlich“ oder zumindest „möglich“ [12]. In einigen Fällen wurden aber neben Grüntee weitere pflanzliche Produkte konsumiert, die ihrerseits mit Leberschäden assoziiert sind (z.B. Cassia angustofolia, Ephedra sinica). Auch existieren experimentelle und klinische Berichte, die auf insgesamt positive Effekte von Grüntee und seinen Extrakten auf Leberschäden und Gesamtmortalität hinweisen.

\section{Klinische Relevanz}

Bei den meisten Präparaten ist der toxische Mechanismus unklar. Die Leberschäden können gravierend sein und in seltenen Fällen zum Leberversagen führen.

\begin{tabular}{|c|c|c|c|}
\hline Produkt / Wirkstoff & Anwendungsbereich & Leberschaden & Schädigungsmechanismus \\
\hline $\begin{array}{l}\text { Mehrere Herbalife }{ }^{\circledR} \\
\text { Kombinationen }\end{array}$ & $\begin{array}{l}\text { Gewichtsreduktion, } \\
\text { Nahrungsergänzungsmittel }\end{array}$ & $\begin{array}{l}\text { Variabel (akute und chronische } \\
\text { Hepatitis, Cholestase, Zirrhose, } \\
\text { Leberversagen) }\end{array}$ & $\begin{array}{l}\text { Nicht bekannt; Autoimmunität? } \\
\text { Kontamination? }\end{array}$ \\
\hline $\begin{array}{l}\text { Usninsäure } \\
\left(\text { z. B. LipoKinetix }{ }^{\circledR}\right)\end{array}$ & Gewichtsreduktion & Akute Hepatitis & Entkopplung der Atmungskette? \\
\hline Hydroxycut $^{\circledR}$ & $\begin{array}{l}\text { Gewichtsreduktion, } \\
\text { Bodybuilding }\end{array}$ & $\begin{array}{l}\text { Akute und/oder cholestatische } \\
\text { Hepatitis, Leberversagen }\end{array}$ & Nicht bekannt \\
\hline Anabolische Steroide & Bodybuilding, Doping & $\begin{array}{l}\text { Cholestase, Steatose, Peliosis } \\
\text { hepatis }\end{array}$ & $\begin{array}{l}\text { Hemmung biliärer Transporter- } \\
\text { systeme }\end{array}$ \\
\hline $\begin{array}{l}\text { Grüner Tee (Camellia } \\
\text { sinensis) }\end{array}$ & Gewichtsreduktion & Akute Hepatitis & $\begin{array}{l}\text { Oxidativer Stress durch } \\
\text { (-)-Epigallocatechin-Gallat? }\end{array}$ \\
\hline Oxy Elite Pro & Gewichtsred., Bodybuilding & Akute und fulminante Hepatitis & Nicht bekannt \\
\hline Ma-Huang (Ephedra sp.) & Gewichtsreduktion & Akute Hepatitis & $\begin{array}{l}\text { Nicht bekannt; Risiko bei } \\
\text { C282Y / H63D Heterozygotie? }\end{array}$ \\
\hline $\begin{array}{l}\text { „Onshidou-Genbi- } \\
\text { Kounou“, „Chaso” } \\
\text { (Japanisches Kraut) }\end{array}$ & Gewichtsreduktion & Akute und fulminante Hepatitis & $\begin{array}{l}\text { Hepatozelluläre Apoptose durch } \\
\text { N-Nitroso-Fenfluramin }\end{array}$ \\
\hline $\begin{array}{l}\text { Germander } \\
\text { (Teucrium chamaedrys) }\end{array}$ & Gewichtsreduktion & $\begin{array}{l}\text { Akute und fulminante Hepatitis, } \\
\text { chronische fibrosierende Hepatitis }\end{array}$ & $\begin{array}{l}\text { Hepatozelluläre Apoptose durch } \\
\text { Neo-Clerodan-Diterpenoide }\end{array}$ \\
\hline
\end{tabular}




\section{Nahrungsergänzungsmittel zur Gewichtsreduktion}

Ein zunehmendes Gesundheitsbewusstsein, aber auch Gewichtsprobleme von vielen Menschen in westlichen Industrienationen haben $\mathrm{zu}$ einem Boom an Produkten zur Unterstützung von Gewichtsreduktion geführt. Diese werden nicht als Medikamente vermarktet, daher weniger kontrolliert und sind meist frei verkäuflich. Einige Produkte sind in jüngerer Zeit mit relevanten Leberschäden in Verbindung gebracht worden ( $\checkmark$ Tab. 2). So wurden Herbalife ${ }^{\mathrm{TM}}$-Produkte in mehreren Ländern Europas und in Israel als Ursache von Leberschäden bestätigt bzw. als wahrscheinliche oder mögliche Auslöser gemeldet [13]. Meist waren mehrere Herbalife ${ }^{\mathrm{T}}$-Produkte eingenommen worden, sodass der eigentliche Auslöser nicht identifiziert werden konnte. In einigen Produkten wurde jedoch eine bakterielle Kontamination festgestellt, die als möglicher Mechanismus denkbar ist.

Andere Nahrungsergänzungsmittel enthielten Usninsäure, die aus Flechtenarten der Gattung der Bartflechten gewonnen wird. Usninsäure hemmt die oxidative Phosphorylierung in Mitochondrien und kann somit theoretisch den Kalorienverbrauch steigern und Gewichtsreduktion fördern. In den USA löste das Nahrungsergänzungsmittel LipoKinetix ${ }^{\circledR}$, welches u. a. Usninsäure enthielt, mehrere Fälle schwerer Leberschäden mit Leberversagen und Lebertransplantation aus. Die amerikanische Food and Drug Administration (FDA) entzog dem Produkt daraufhin die Zulassung [14].

Hydroxycut $^{\circledR}$ und OxyELITE ${ }^{\circledR}$ waren in den USA zur Unterstützung von Gewichtsabnahme und bei Bodybuildern weit verbreitet. Hydroxycut ${ }^{\circledR}$ enthielt Garcinia cambogia, Gymnema sylvestre und Grüntee. Der Wirkstoff in OxyELITE ${ }^{\circledR}$ war 1,3-Dimethylamylamin, das auch in zahlreichen anderen Nahrungsergänzungsmitteln enthalten war. Bei Hydroxycut ${ }^{\circledR}$ kam es zu akuten oder eher subakuten cholestatischen Verläufen mit Remission bei Absetzen [15]. Im Falle von OxyELITE ${ }^{\circledR}$ kam es sogar zu einem Todesfall und 4 Lebertransplantationen [16], sodass beide Produkte und andere 1,3-Dimethylamylamin-haltige Präparate verboten wurden.

\section{Klinische Relevanz}

Nahrungsergänzungsmittel zur

Gewichtsreduktion stehen momentan

unter besonderer Beobachtung
Literatur

1 Eardley S, Bishop FL, Prescott P et al. A systematic literature review of complementary and alternative medicine prevalence in EU. Forsch Komplementmed 2012; 19 (Suppl. 2): 18-28

2 Eisenberg DM, Davis RB, Ettner SL et al. Trends in alternative medicine use in the United States, 1990-1997: results of a follow-up national study. JAMA 1998; 280: 1569-1575

3 Vlietinck A, Pieters L, Apers S. Legal requirements for the quality of herbal substances and herbal preparations for the manufacturing of herbal medicinal products in the European Union. Planta Med 2009; 75: 683-688

4 Navarro VJ, Barnhart H, Bonkovsky HL et al. Liver injury from Herbals and Dietary Supplements in the US Drug Induced Liver Injury Network. Hepatology 2014; 60: 1399-1408

5 Aithal GP, Rawlins MD, Day CP. Clinical diagnostic scale: a useful tool in the evaluation of suspected hepatotoxic adverse drug reactions. J Hepatol 2000; 33: 949-952

6 Rochon J, Protiva P, Seeff LB et al. Drug-induced Liver Injury Network (DILIN): Reliability of the Roussel Uclaf Causality Assessment Method for assessing causality in drug-induced liver injury. Hepatology 2008; 48: 1175-1182

7 DeLeve LD, Shulman HM, McDonald GB. Toxic injury to hepatic sinusoids: sinusoidal obstruction syndrome (veno-occlusive disease). Semin Liver Dis 2002; 22: 27-42

8 Teschke R, Wolff A, Frenzel C, Schulze J. Review article: Herbal hepatotoxicity - an update on traditional Chinese medicine preparations. Aliment Pharmacol Ther 2014; 40: 32-50

9 Fau D, Lekehal M, Farrell G et al. Diterpenoids from germander, an herbal medicine, induce apoptosis in isolated rat hepatocytes. Gastroenterology 1997; 113: 1334-1346

10 European Medicines Agency. Public statement on Chelidonium majus L., herba. http://www.ema. europa.eu/docs/en_GB/document_library/Public statement/2012/01/WC500120714.pdf. Zugriff: 27.5.2015

11 Stickel F, Baumüller HM, Seitz KH et al. Hepatitis induced by Kava-Kava (Piper methysticum rhizoma). J Hepatol 2003; 39: 62-67

12 Sarma DN, Barrett ML, Chavez ML et al. Safety of green tea extracts. A systematic Review by the US Pharmacopeia. Drug Saf 2008; 31: 469-484

13 Stickel F, Kessebohm K, Weimann R, Seitz HK. Review of Liver Injury Associated with Dietary Supplements. Liver Int 2011; 31: 595-605

14 U.S. Food and Drug Administration. Lipokinetix. www.fda.gov/Safety/MedWatch/Safetylnformation/ SafetyAlertsforHumanMedicalProducts/ ucm172824.htm. Zugriff: 13.5.2015

15 U.S. Food and Drug Administration. Recall - Firm Press Release. www.fda.gov/Safety/recalls/ ucm145164.htm. Zugriff: 13.5.2015

16 Roytman MM, Pörzgen P, Lee CL et al. Outbreak of severe hepatitis linked to weight-loss supplement OxyELITE Pro. Am J Gastroenterol 2014; 109: 1296-1298

17 Seeff LB, Bonkovsky HL, Navarro VJ, Wang G. Herbal products and the liver: a review of adverse effects and mechanisms. Gastroenterology 2015; 148: 517-532

18 Stickel F, Shouval D. Hepatotoxicity of herbal and dietary supplements: an update. Arch Toxicol 2015, DOI 10.1007/s00204-015-1471-3

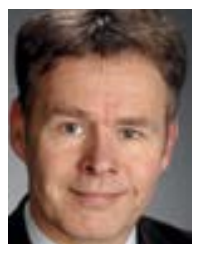

PD Dr. med. Felix Stickel ist Leiter der Forschungsgruppe Experimentelle Hepatologie der Abteilung für Gastroenterologie und Hepatologie, Universitätsspital Zürich, Schweiz und Leiter des Hepatologieambulatoriums, Klinik Beau-Site, Hirslanden Bern AG, Schweiz. Felix.stickel@hirslanden.ch

Anmerkung

Für eine detaillierte

Beschreibung der

Thematik wird auf zwei aktuelle Übersichtsarbeiten hingewiesen $[17,18]$.

Interessenkonflikt

Der Autor gibt an, dass kein Interessenkonflikt besteht.

DOI 10.1055/s-0041-102437

Dtsch Med Wochenschr 2015; 140: 908-911

(c) Georg Thieme Verlag KG . Stuttgart - New York . ISSN 0012-0472 